\title{
Diploid males support a two-step mechanism of endosymbiont-induced thelytoky in a parasitoid wasp
}

Wen-Juan Ma ${ }^{1,2^{*}}$, Bart A. Pannebakker ${ }^{3}$, Louis van de Zande ${ }^{1}$, Tanja Schwander ${ }^{1,2}$, Bregje Wertheim ${ }^{1}$ and Leo W. Beukeboom ${ }^{1}$

\begin{abstract}
Background: Haplodiploidy, where females develop from diploid, fertilized eggs and males from haploid, unfertilized eggs, is abundant in some insect lineages. Some species in these lineages reproduce by thelytoky that is caused by infection with endosymbionts: infected females lay haploid eggs that undergo diploidization and develop into females, while males are very rare or absent. It is generally assumed that in thelytokous wasps, endosymbionts merely diploidize the unfertilized eggs, which would then trigger female development.

Results: We found that females in the parasitoid wasp Asobara japonica infected with thelytoky-inducing Wolbachia produce $0.7-1.2 \%$ male offspring. Seven to $39 \%$ of these males are diploid, indicating that diploidization and female development can be uncoupled in A. japonica. Wolbachia titer in adults was correlated with their ploidy and sex: diploids carried much higher Wolbachia titers than haploids, and diploid females carried more Wolbachia than diploid males. Data from introgression lines indicated that the development of diploid individuals into males instead of females is not caused by malfunction-mutations in the host genome but that diploid males are most likely produced when the endosymbiont fails to activate the female sex determination pathway. Our data therefore support a two-step mechanism by which endosymbionts induce thelytoky in A. japonica: diploidization of the unfertilized egg is followed by feminization, whereby each step correlates with a threshold of endosymbiont titer during wasp development.
\end{abstract}

Conclusions: Our new model of endosymbiont-induced thelytoky overthrows the view that certain sex determination mechanisms constrain the evolution of endosymbiont-induced thelytoky in hymenopteran insects. Endosymbionts can cause parthenogenesis through feminization, even in groups in which endosymbiont-diploidized eggs would develop into males following the hosts' sex determination mechanism. In addition, our model broadens our understanding of the mechanisms by which endosymbionts induce thelytoky to enhance their transmission to the next generation. Importantly, it also provides a novel window to study the yet-poorly known haplodiploid sex determination mechanisms in haplodiploid insects.

Keywords: Diploid males, Diploidization, Endosymbiont, Feminization, Haplodiploidy, Sex determination, Thelytoky, Wolbachia titer

\footnotetext{
* Correspondence: wenjuanma84@gmail.com

${ }^{1}$ Evolutionary Genetics, Groningen Institute for Evolutionary Life Sciences,

University of Groningen, Groningen, The Netherlands

${ }^{2}$ Department of Ecology and Evolution, University of Lausanne, Lausanne,

Switzerland

Full list of author information is available at the end of the article
}

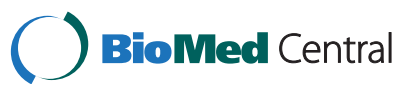

(C) 2015 Ma et al.; licensee BioMed Central. This is an Open Access article distributed under the terms of the Creative Commons Attribution License (http://creativecommons.org/licenses/by/4.0), which permits unrestricted use, distribution, and reproduction in any medium, provided the original work is properly credited. The Creative Commons Public Domain Dedication waiver (http://creativecommons.org/publicdomain/zero/1.0/) applies to the data made available in this article, unless otherwise stated. 


\section{Background}

Although sexual reproduction is ubiquitous in nature, approximately two percent of described insect species reproduce parthenogenetically $[1,2]$, including cyclical parthenogens (e.g., aphids and cynipids), facultative parthenogens, as well as obligate parthenogens. Thelytoky is a form of parthenogenesis in which unfertilized eggs develop into females. In insects, thelytokous reproduction can be determined by the host's nuclear genome (e.g., [3-6]), but also by intracellular endosymbionts such as Wolbachia, Cardinium and Rickettsia (e.g., [7-9]). Endosymbionts are vertically transmitted via the egg cytoplasm and therefore not transmitted by males, thelytoky-induction is adaptive for the endosymbionts because it enhances their transmission rate. Endosymbiont-induced thelytoky has been mainly documented in haplodiploid species (reviewed in [10-12]). Under haplodiploidy, females are diploid and develop from fertilized eggs while haploid males develop from unfertilized eggs [2,13]. Endosymbiont-infected females in these species lay unfertilized eggs that undergo diploidization and develop into females, but how these processes are induced and regulated remains enigmatic.

The simplest explanation of how endosymbionts could induce thelytoky in haplodiploids is that they cause haploid eggs to undergo diploidization. These diploidized eggs would then develop into females as a consequence of the host's haplodiploid sex determination system $[7,8$, 10, 14-18]. However, a recent study by Giorgini et al. [9] found that curing the parasitoid wasp Encarsia formosa of its thelytoky-inducing Cardinium with antibiotics resulted in progenies consisting entirely of diploid males, rather than haploid males as expected under haplodiploidy. A likely explanation is that Cardinium in this species induces the feminization of diploid embryos, whereas the diploidization of unfertilized eggs is controlled by the host's genome [9]. Diploid males were also detected in the progenies of infected thelytokous Trichogramma wasps that had lowered Wolbachia titer following heat treatment [19]. These two studies suggest that female development under endosymbiont-induced thelytoky is not necessarily attained through diploidization alone, and that feminization may not be a direct consequence of diploidization.

Here, we investigate the causes of the frequent and spontaneous occurrence of males (haploid and diploid) in Wolbachia-infected thelytokous populations of the parasitoid wasp Asobara japonica [20, 21], and use this information to propose a model for Wolbachia-induced thelytokous reproduction. First, we quantified the frequency of haploid and diploid males among four thelytokous strains. Next, we examined the effect of the Wolbachia titer of parental females on the frequency and ploidy of males among their progenies, because
Wolbachia dosage-dependent effects have been implicated in male production in other thelytokous species (e.g., [22-25]). We further assessed the association between Wolbachia titer and ploidy and sex of individual wasps. Finally, we introgressed alleles from a thelytokous into a sexual strain, to test whether the production of diploid males could be due to malfunction-mutations in the thelytokous genome for the female sex determination pathway. Such mutations could render individuals with a thelytokous genome unable to undergo feminization in the absence of the endosymbiont. Long-term infection with endosymbionts can result in a dependency of the host on its symbiont, for processes formerly accomplished by pathways encoded by the host genome (e.g., [26, 27]). Such a dependency may occur for female development in species with endosymbiont-induced thelytoky. Since it is the endosymbiont that triggers female development, females cured of their endosymbionts always produce sons, [14-16, 21]. Our previous study has ruled out complementary sex determination for A. japonica [28], but it is currently not known what its sex determination mechanism is (see Discussion for further details).

Our results suggest that diploidization and feminization are two separate processes required for Wolbachia-induced thelytoky in Asobara japonica, and that these two processes may depend on different Wolbachia titers. The combination of these findings with the studies of Giorgini et al. [9] and Tulgetske [19] led us to formulate a two-step model for the mechanism of endosymbiont-induced thelytoky. We discuss how this model challenges the view that endosymbiont-induced thelytoky can only occur in haplodiploid species with specific molecular mechanisms of sex determination.

\section{Methods}

\section{Wasp cultures and male frequency}

Four Wolbachia-infected thelytokous strains and one uninfected sexual strain of Asobara japonica were used for investigating the causes of male development under thelytoky. All strains were collected in Japan, described in Mitsui et al. [29] and Murata et al. [30], and kindly provided by M.T. Kimura. The sexual strain originated from the island of Amami-oshima (AO), and the four thelytokous strains were originally collected from Sapporo (SPP), Kagoshima (KG), Hirosaki (HR) and Tokyo (TK) on the mainland of Japan. Wasps were cultured on second-instar Drosophila melanogaster larvae as hosts at $25{ }^{\circ} \mathrm{C}$, with a $16 \mathrm{~L}$ : 8D light-dark cycle and $60 \%$ relative humidity (for details see [28]).

To investigate the frequency of male production in different thelytokous strains, three replicates each consisting of five to eight females from all four thelytokous strains were offered approximately 200 second-instar $D$. melanogaster larvae for oviposition. The emerged 
offspring were counted and sexed. This procedure was repeated for five successive generations for the thelytokous KG strain to obtain sufficient sample sizes for the introgression experiment (see below).

\section{Determination of ploidy level}

Flow cytometry was used to determine the ploidy level of males $(n=298)$ among progenies of females from the four thelytokous strains, following methods described by Ma et al. [28]. In short, the head of each individual (freshly killed by freezing at $-20{ }^{\circ} \mathrm{C}$ ) was homogenized in $500 \mu \mathrm{l}$ Galbraith buffer and the DNA was stained with $10 \mu \mathrm{l}$ propidium iodide $(2.5 \mathrm{mg} / \mathrm{ml})$. The total DNA content of approximately 2500 nuclei was measured on a Coulter Epics MXL flow cytometer (Beckman Coulter, Miami, FL, USA), or 10,000 nuclei on BD FACSAria ${ }^{\mathrm{Ta}}$ II Cell Sorting System (Becton Dickinson B.V., Breda, The Netherlands). Flow cytometric DNA histograms of sampled wasp individuals were generated with WinMDI software package (version 2.9, The Scripps Research Institute, La Jolla, CA, USA) or BD FACSDiva ${ }^{\text {Th }}$ (version 6.1.2; see Additional file 1: Figure S1). Two females per strain (collected from mass cultures) were used as diploid controls. Males were classified as haploid or diploid by comparing the mean log-transformed propidium iodide fluorescence (representing DNA amount per nucleus) to the diploid control.

\section{Antibiotic treatment}

To test whether the Wolbachia titer of a parental thelytokous female affects male offspring production, different concentrations of the antibiotic rifampicin (Sigma-Adlrich, St. Louis, USA) were fed to thelytokous HR females. Rifampicin has been shown to have little impact on the development of Asobara wasps [26]. No effect on life-history traits, such as brood size and pupal mortality, was found in our experiments (data not shown). HR thelytokous females were offered host larvae that had fed on a yeast suspension containing different concentrations of rifampicin ranging from 0 (control), $0.00001,0.00005,0.0001,0.001,0.005,0.01,0.05,0.1$ and $1 \mathrm{mg} / \mathrm{g}$ (RIF0-RIF9). Wasp larvae ingest the antibiotics by feeding on the treated hosts and differentiate into females with a reduced endosymbiont titer [26]. Ten females were randomly selected from each antibiotic concentration. Each female was allowed to lay eggs in approximately 50 untreated host larvae, and offspring emerging from these hosts were counted and sexed (data are available at Dryad repository [31]). Wolbachia titers were determined via quantitative PCR (see below) both in the thelytokous mothers and in a randomly collected set of males and females from the mass culture.

\section{Quantitative PCR (qPCR)}

The effect of antibiotic treatment on Wolbachia titer of females (emerged from the treated host larvae) was quantified by $\mathrm{qPCR}$ in three females per applied antibiotic concentration [31]. Similar qPCRs were performed to establish the correlation between Wolbachia titer and ploidy and sex of wasps from the mass culture [31] (females: $\mathrm{n}=18$, haploid males: $\mathrm{n}=12$, diploid males: $\mathrm{n}=$ 12). Genomic DNA was extracted from the whole body of each adult wasp individual, using a high-salt protocol (adjusted from [32]). The Wolbachia titer was determined from the amount of the Wolbachia-specific gatB gene, and two $A$. japonica reference genes, the nuclear ITS2 (internal transcribed spacer) and mitochondrial COI (cytochrome oxidase I subunit, see Additional file 2: Figure S2). The following primers were used (from [20, 33]): gatB-F: 5'-GAA GCA AAG AGG ATG CAA GC3 ' and gatB-R: 5' -TCC TGG CTT ACC TCA ACA GG3', producing a 73-bp amplicon; ITS2-F: $5^{\prime}$-GGC AAG CAC AAT CAA GGT CT-3' and ITS2-R: 5' -ACA AAA ACA AAT TTT GCG GC-3', producing a 93-bp amplicon; COI-F: 5 '-ACC TGT AAT ATT AGG TGG ATT TGG-3' and COI-R: 5' -CCA ACA CCT ACA TTT AAT ATT CCT CT-3', producing a 139 bp amplicon. Each qPCR was performed in a total volume of $25 \mu \mathrm{l}$, containing 1× SybrGreen Mastermix (Warrington, Cheshire, UK), 400nM forward primer, 400nM reverse primer and $2 \mu \mathrm{l}$ DNA template. The Applied Biosystems 7300 RealTime PCR System (Applied Biosystems Inc., Singapore) was used for all qPCR reactions. qPCR conditions for all genes were as follows: $15 \mathrm{~min}$ at $95^{\circ} \mathrm{C}$, then 40 cycles of $25 \mathrm{~s}$ at $95{ }^{\circ} \mathrm{C}, 45 \mathrm{~s}$ at $52{ }^{\circ} \mathrm{C}$ and $1 \mathrm{~min}$ at $72{ }^{\circ} \mathrm{C}$, and finally $7 \mathrm{~min}$ at $72^{\circ} \mathrm{C}$.

The qPCR data were analysed with the LinRegPCR software (version 2012.3.2.0, $[34,35]$ ) to calculate the starting concentrations $\left(\mathrm{N}_{0}\right)$ of the target and the reference genes [36]. The efficiency of primers and PCR was optimized prior to the qPCR. The relative quantity of $g a t B$ was established as the ratio (R) of the starting concentrations of the focal gene $\left(\mathrm{N}_{0 \_}\right.$gatB $)$to the geometric mean of the reference genes $\left(\mathrm{N}_{0}\right.$ COI\&ITS2): $\mathrm{R}=\mathrm{N}_{0 \_}$gatB $/ \mathrm{N}_{0 \_} C O I \& I T S 2$.

\section{Introgression experiment}

To test whether individuals with a thelytokous genome were unable to undergo feminization in the absence of the endosymbiont, alleles of the thelytokous KG strain were introgressed into the sexual AO genome (Fig. 1). $\mathrm{AO}$ females that were mated with $\mathrm{AO}$ males served as a sexual control (data from [21]). The introgression started by setting up crosses between males from the KG strain with females from the sexual AO strain for $24 \mathrm{~h}$. Spontaneously occurring asexual KG males may be haploid or diploid. Diploid males were shown to be fertile, 


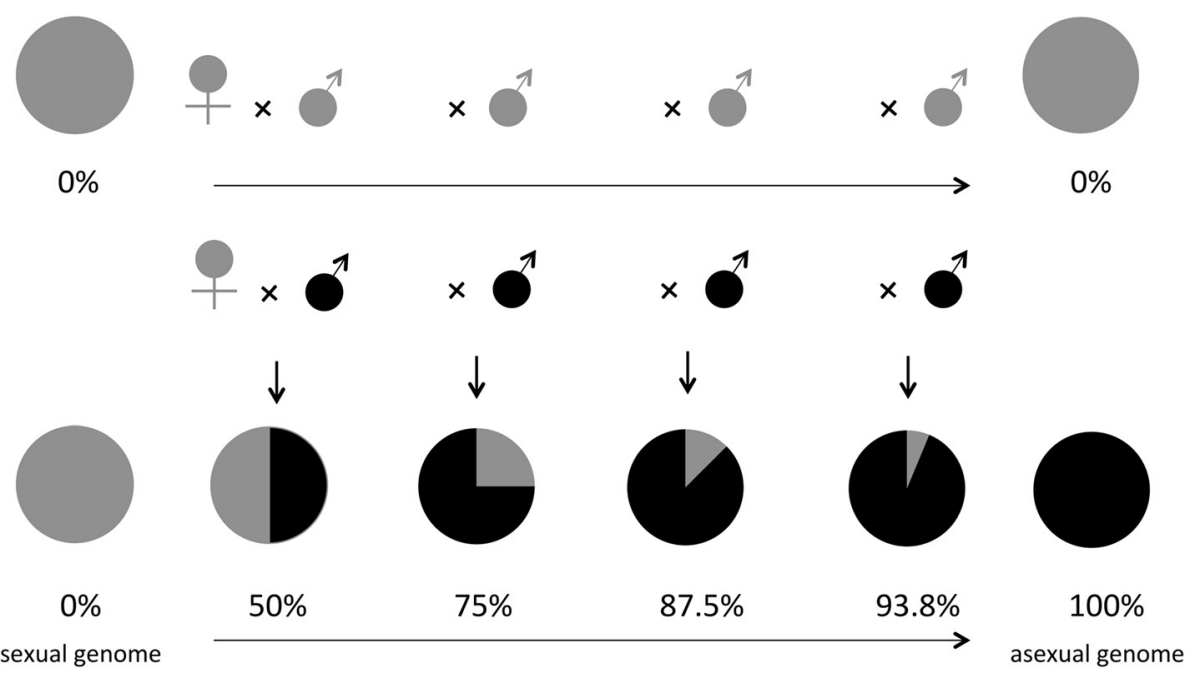

Fig. 1 Introgression experiment. Sexual females were backcrossed to asexual males for four generations. Grey \& black symbols depict sexual and asexual genotypes respectively. Pie charts show the relative asexual composition of the female genome for progressive generations of introgression

although they produce a lower number of female offspring (triploid) than haploid males [37]. As triploid females were found to be sterile, we only selected haploid KG males as fathers in the introgression experiment (the ploidy of males was determined after the matings and matings involving diploid males were discarded). Sixty sexual females mated to haploid asexual KG males were subsequently placed in a glass bottle with agar medium and a layer of $1.5 \mathrm{ml}$ yeast solution $(0.4 \mathrm{~g} / \mathrm{ml})$ containing approximately 100 second-instar host larvae for oviposition during $36 \mathrm{~h}$ (for details see [28]). After approximately 13 days, male offspring emerged and were collected (males emerge earlier than females). The remaining wasp pupae were individually isolated in vials to obtain virgin hybrid females. These F1 hybrid females were backcrossed to a thelytokous KG male (with haploidy verified as described above). This experimental procedure was repeated for four generations, yielding females with $50 \%$ (hybrid introgression generation 1, F1), $75 \%$ (backcross introgression generation 2, G2), $87.5 \%$ (G3), and $93.8 \%$ (G4) of the thelytokous genome (Fig. 1). Brood sizes and the proportions of female offspring were scored per generation (due to logistic reasons, phenotyping of the G3 generation was not possible and therefore no data are provided), with sexual $\mathrm{AO}$ females mated with $\mathrm{AO}$ males serving as a control [31] (data from [21]). As the proportion of hybrid females that produced daughters (i.e., were still able to mate and fertilize their eggs) decreased during successive introgression generations [21], the number of broods with at least one daughter that could be used for offspring sex ratio analysis decreased gradually. Thus, the number of broods used for sex ratio analysis decreased from 60 in the cross between sexual females to KG males, to 8 in the F1 generation, to 8 in G2 and 4 in G4. The ploidy of 51 randomly selected male offspring produced during F1 generation was analysed by flow cytometry (as described above). The ploidy of male offspring from subsequent generations was not analysed, because offspring sex ratios in these generations indicated that the ratio of diploid to haploid males did not vary (see Results for details).

\section{Statistical analyses}

A generalized linear model (glm) was used to test possible effects of antibiotic treatment of parental females on male offspring production. Antibiotic concentration was used as a quantitative explanatory variable and the proportion of male offspring as the response variable (weighted by the brood size via the logit function), with a quasi-binomial error structure to correct for overdispersion [38]. To compare diploid male numbers between antibiotic treatments, a glm was used in which the number of diploid males was the response variable and antibiotic concentration a qualitative explanatory variable with a log link function and a quasi-poisson error structure to correct for over-dispersion [38]. Multiple comparisons of diploid male numbers among different antibiotic treatments were performed with the Tukey test, as implemented in the R package multcomp for general linear hypotheses [39].

A glm was used to compare the Wolbachia titer among females of the various antibiotic treatments, measured as the gene quantity of gatB relative to the endogenous controls. As Wolbachia titers were normally distributed, this glm used an identity link function and a gaussian error structure. Wolbachia titer was used as the response variable and the antibiotic concentration as a quantitative 
explanatory variable. To test whether Wolbachia titer differed between individuals with different ploidy levels, diploids (females and males) were compared with haploid males using a Wilcoxon rank-sum test. The Wolbachia titer between diploid females and diploid males was analysed in a similar way.

Glms were also used to compare brood sizes and daughter proportions among introgression generations, with the percentage of the thelytokous genome as a quantitative explanatory variable. Brood size was modeled as the response variable with a quasi-poisson error structure to correct for over-dispersion, and proportion of female offspring as the response variable with a quasibinomial error structure to correct for over-dispersion [38]. To check for the possibility of hybrid breakdown causing male mortality during the introgression experiment, male offspring numbers from virgin sexual and virgin introgressed F1 (50 \% sexual-50 \% thelytokous) females were compared with a glm model. In this glm, female genotype is the explanatory variable and the male offspring number the response variable with a quasi-poisson error structure to correct for overdispersion [38].

\section{Results}

\section{Frequency and ploidy of males}

Males were found at frequencies of $0.7 \%, 1.2 \%$ and $1.2 \% \quad(\mathrm{n}=$ approximately 2,500 offspring per strain) among the progenies of females from the thelytokous strains SPP, HR and TK, respectively. Similarly, a total of 220 males $(1.2 \%)$ were found in the KG strain using a much larger sample size (18,000 offspring; Table 1). These male frequencies in thelytokous $A$. japonica are similar to the ones reported by Reumer et al. [20]. Flow cytometry revealed that $73 \%$ of these males (218 out of 298) were haploid and the remaining $27 \%$ (80 out of 298 ) were diploid, with proportions of diploid males ranging from 7-39 \% among different thelytokous strains (Table 1).

\section{Effect of Wolbachia titer}

To investigate whether male production depended on Wolbachia titer of parental females, thelytokous females were offered host larvae that had been fed different antibiotic concentrations. The vast majority of emerged wasps from these antibiotics-treated hosts were females (approximately $98.7 \%$ ). For each antibiotic treatment, ten females were randomly selected and were then offered untreated host larvae for oviposition and their offspring were counted and sexed. The proportion of male offspring (haploid and diploid combined) gradually increased with higher concentrations of antibiotics administered to the hosts, ranging from $0 \%$ to approximately $60 \%\left(\mathrm{glm}, \mathrm{F}_{1,91}=158.88, P<0.0001\right.$, Fig. 2). Wolbachia titer in mothers was verified by qPCR and revealed that higher antibiotic concentrations indeed resulted in lower Wolbachia titers (glm, $\mathrm{F}_{1,19}=63.70, P<0.0001$; Fig. 3). Diploid males occurred at very low frequencies among the progeny of all antibiotic-treated thelytokous females, and there was no significant difference among antibiotic treatments in the number of diploid males $\left(\mathrm{glm}, \mathrm{F}_{9,91}=\right.$ $0.916, P=0.341$; Table 2).

We then assessed the association between Wolbachia titer and ploidy, and between Wolbachia titer and the sex of thelytokous offspring: haploid males, diploid males and diploid females. All tested diploid females and diploid males were infected by Wolbachia. By contrast, only 7 out of 12 of haploid males were infected. The Wolbachia titer of diploid individuals (females and males pooled) was significantly higher than that of haploid males (Wilcoxon test, $W=5, P<0.0001$; Fig. 4), suggesting that a minimum Wolbachia titer is required to cause diploidization of unfertilized haploid eggs. Moreover, among the diploid individuals, the Wolbachia titer of females was significantly higher than that of males (Wilcoxon test, $W=59, P=0.0088$; Fig. 4), further suggesting an additional role for Wolbachia titer in the feminization of diploid embryos.

\section{Wolbachia effect on host sex determination}

We hypothesized that if the thelytokous host's female sex determination pathway became dependent on Wolbachia, replacing the sexual genome by the thelytokous genome in the absence of Wolbachia may cause diploid fertilized eggs to develop into males rather than females. Upon introgression, diploid males are expected to develop from fertilized eggs of females from the first introgression generation onward if putative malfunctionmutations in the female sex determination pathway are dominant, and if such mutations are recessive, an

Table 1 Number and ploidy level of males in progenies of thelytokous females of four different A. japonica strains

\begin{tabular}{lllll}
\hline Line & No. haploid males & No. diploid males & No. total males & Prop. diploid males \\
\hline SPP & 11 & 7 & 18 & $39 \%$ \\
KG & 153 & 67 & 220 & $30 \%$ \\
HR & 26 & 4 & 30 & $13 \%$ \\
TK & 28 & 2 & 30 & $7 \%$ \\
Total & 218 & 80 & 298 & $27 \%$ \\
\hline
\end{tabular}




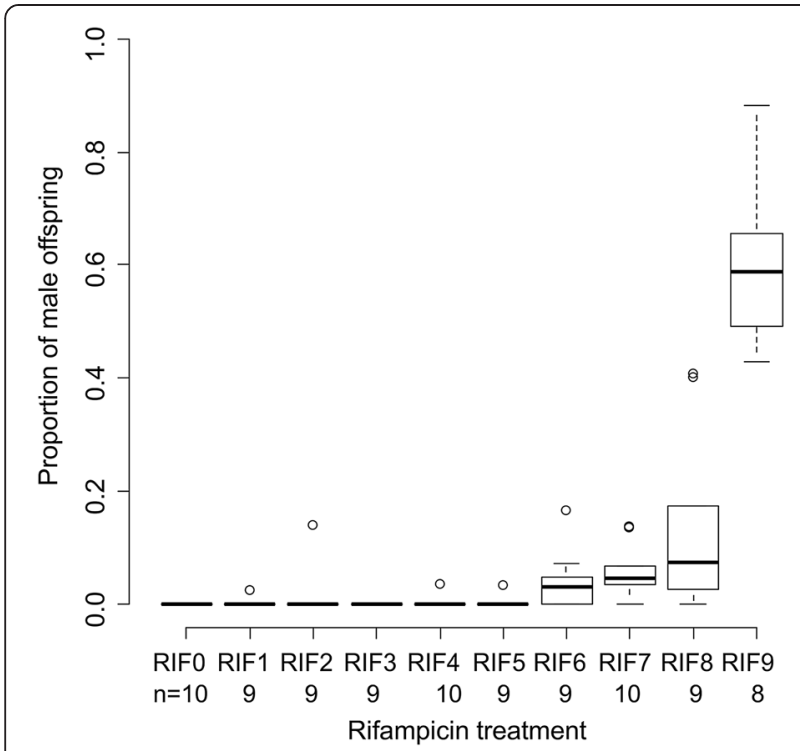

Fig. 2 Proportion of males among progenies of thelytokous HR strain females treated with different concentrations of rifampicin (RIF). RIF0 is the no antibiotics treatment control, RIF1 to RIF9 depict the series of different concentrations of rifampicin $(0.00001,0.00005$, $0.0001,0.001,0.005,0.01,0.05,0.1$ and $1 \mathrm{mg} / \mathrm{g}$ respectively). Sample sizes $(n)$ are given for each treatment

increasing proportion of diploid males is expected when more thelytokous alleles are introgressed. The production of diploid sons would then generate a corresponding decrease of the proportion of daughters, since some fraction of fertilized eggs would develop into diploid males instead of females. However, we did not find an increase in diploid males, or a decrease in the production of daughters, across introgression generations. Not a single diploid individual was found in a random sample of 51 males from the F1 introgression generation and daughter production increased across introgression generations. Females of generations F1 and G2 produced a higher proportion of daughters than sexual controls when mated with a male from the thelytokous KG strain (glm, F1 and G2: $P<0.009$; Fig. 5), and a higher proportion of female offspring was also found when these females had mated with sexual AO males (glm, Turkey contrast, all $P<0.001$ ). These high proportions of female offspring are unlikely to stem from mortality of males as a consequence of hybrid breakdown because all-male broods of virgin sexual females and F1 introgressed females were equal in size $(82.3 \pm 2.2$ versus $82.0 \pm 3.0$ males; glm, $\left.\mathrm{F}_{1,139}=0.006, P=0.94\right)$. In addition, females of generation G4 with the highest representation of the thelytokous genome (estimated $93.8 \%$ ) produced a similar proportion of daughters as the control sexual cross (glm, $\mathrm{t}=-0.172, P=0.863$ ). These combined data provide evidence that the host sex-determination pathway did not become dependent on Wolbachia, and is not responsible for diploid male occurrence in the asexual strain.

\section{Discussion}

Males are rare or absent in the majority of thelytokous species (reviewed in [40]). However, in agreement with previous reports $[20,33]$, we observed the frequent occurrence of males (0.7-1.2\%) in four thelytokous strains of the parasitoid wasp A. japonica. We found that a significant proportion (7-39\%) of these males are diploid rather than haploid as would be expected given their sex determination mechanism. We investigated the possible

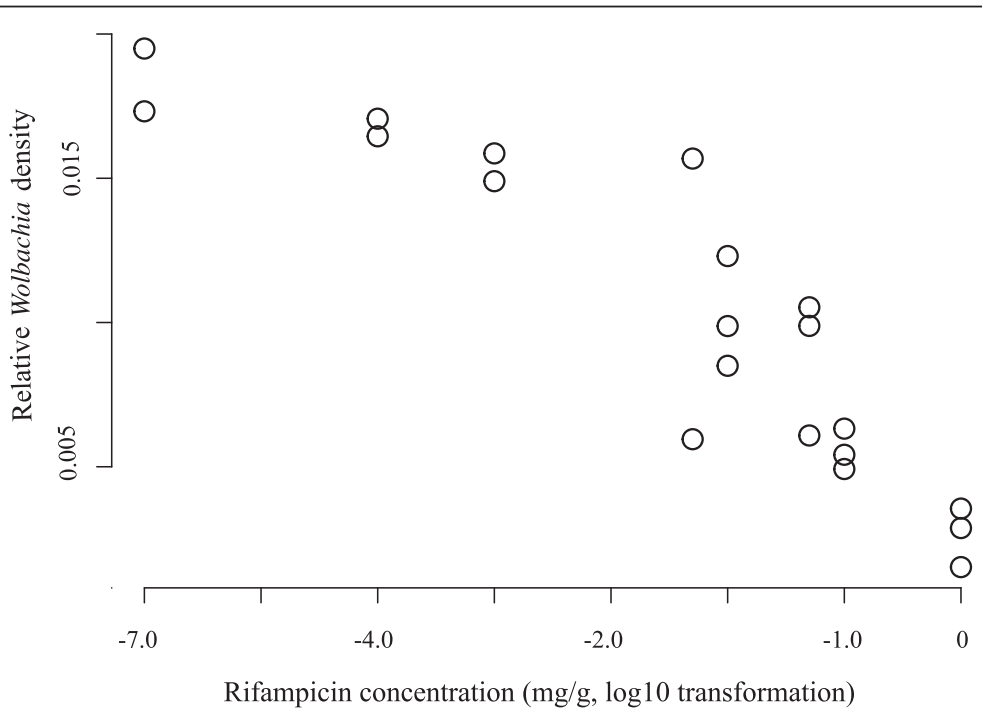

Fig. 3 Wolbachia titer in parental females as a function of rifampicin (RIF) treatment. Wolbachia titer is determined by qPCR of the gatB gene relative to the reference genes $\mathrm{COI}$ and ITS2, and depicted along the x-axis as log transformed values 
Table 2 Number of haploid and diploid males among progenies of thelytokous HR strain females treated with different antibiotic concentrations

\begin{tabular}{lllllllllll}
\hline Treatment & RIF0 & RIF1 & RIF2 & RIF3 & RIF4 & RIF5 & RIF6 & RIF7 & RIF8 & RIF9 \\
\hline Haploid males & 0 & 2 & 4 & 0 & 0 & 1 & 6 & 13 & 13 & 21 \\
Diploid males & 0 & 0 & 0 & 0 & 1 & 0 & 0 & 2 & 1 \\
Total tested & 0 & 2 & 4 & 0 & 1 & 1 & 6 & 14 & 14 & 22 \\
\hline
\end{tabular}

RIF = rifampicin, see text for concentrations

causes for this high occurrence of males (both haploid and diploid) to develop insights into how Wolbachia induces thelytoky in its host.

The simplest mechanism for endosymbiont-induced thelytoky in species with haplodiploid sex determination is that all-female progenies are the result of diploidization of unfertilized haploid eggs through the action of the endosymbiont (e.g., [14, 15, 41]). Diploid eggs would then develop into females following the host's sex determination system. However, the spontaneous occurrence of diploid males in the progeny of thelytokous females (e.g., [19], this study), as well as upon removal of the endosymbiont [9] suggests that the mechanisms of thelytoky induction by endosymbionts may be more complex (i.e., involve feminization in addition to diploidization). Occasional development of haploid males in thelytokous strains has been considered to be the result of stochastic losses of the bacteria, (partial) failure of endosymbiont reproductive manipulation, or maladaptation between two co-evolved parties [20, 42]. However, how diploid males can arise in the broods of thelytokous females has thus far remained unexplained.

One possible explanation for the diploid male occurrence is that female sex determination has become dependent on the endosymbiont in thelytokous $A$. japonica. This has been observed in the lepidopterans Ostrinia scapulalis [43] and Eurema mandarina [44]. However, our introgression of alleles from a thelytokous into a sexual strain did not result in any diploid males, but led to higher proportions of females among the progenies in multiple generations of introgression. These results indicate that diploid males are not produced because of a defective female sex determination pathway in the thelytokous genomes. This implies that diploid

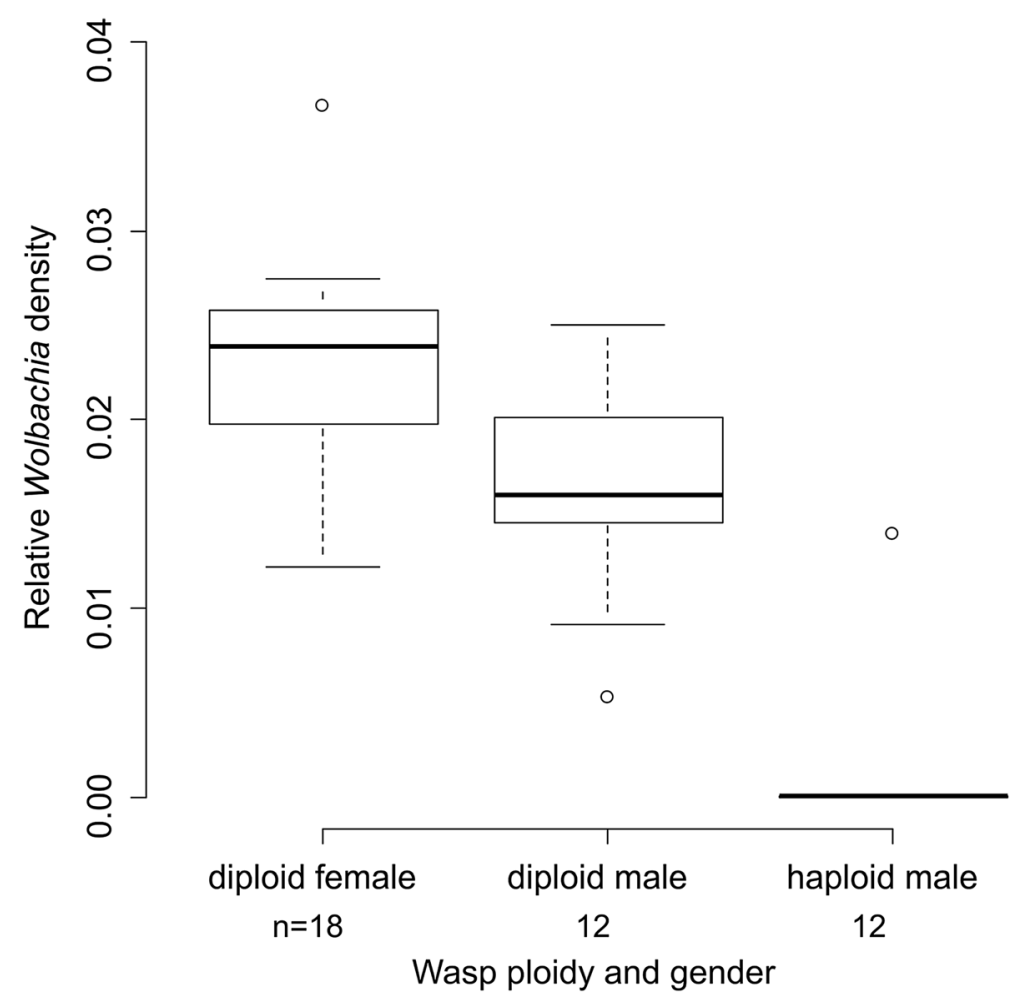

Fig. 4 Wolbachia titer in diploid females, diploid males and haploid males in the untreated thelytokous KG strain. Wolbachia titer is determined by qPCR of the gatB gene relative to the reference genes COI and ITS2. Sample sizes (n) are given for each treatment 


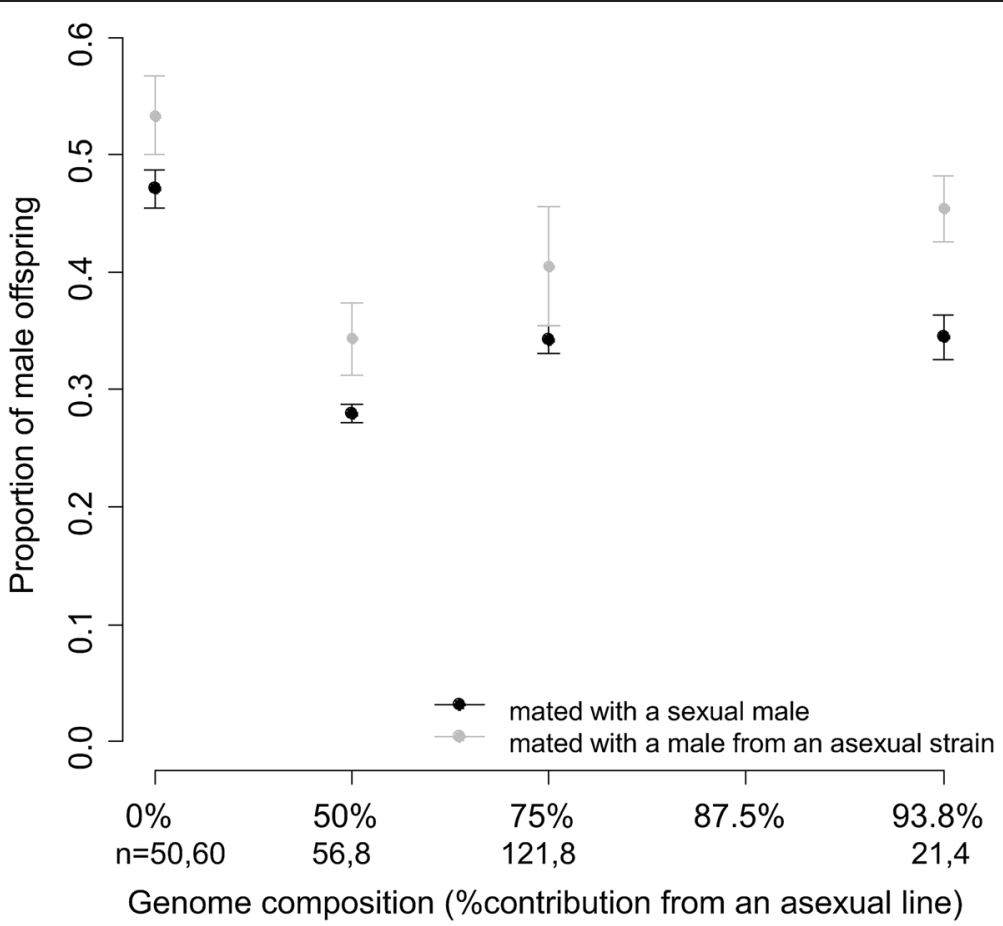

Fig. 5 The proportion of male offspring in progenies of females with at least one daughter (i.e., females producing only sons are excluded) for different categories of sexual-asexual hybrid females and pure sexual females (100\% sexual genome). Females are mated with males from the thelytokous KG (in grey) or the sexual AO strain (in black, as a control, data from Ma et al. [14]). Bars indicate standard errors, $n$ indicates the sample size for each generation and F1, G2, and G4 refer to the progressive introgression generations

males are most likely produced when Wolbachia fails to properly manipulate host reproduction.

We found an effect of Wolbachia titer in parental females on male production (haploids and diploids pooled), as exposing females to increasing antibiotic concentrations resulted in an increased production of males. Similar dosage-dependent effects of Wolbachia were found on male production in the thelytokous parasitoid Muscidifurax uniraptor [23], on cytoplasmic incompatibility in Drosophila [22], and on feminization in the leafhopper Zyginidia pullula [24] and the mosquito Aedes aegypti [25]. However, we did not find a similar Wolbachia dosage effect in parental females on the production of diploid males, although the probability of detecting such an effect was low given that we found few diploid males overall. A putative correlation between a mother's Wolbachia titer and her propensity to produce diploid sons may also be weak because of the stochasticity in Wolbachia titers in individual eggs. Consistent with this view, we did find a correlation at the individual level between Wolbachia titer and wasp ploidy. Haploid males were often not infected at all, or, when infected, they carried a much lower Wolbachia titer than diploid individuals. This suggests that a minimal Wolbachia titer is required for the diploidization of unfertilized haploid eggs. Furthermore, diploid females carried significantly more Wolbachia than diploid males, suggesting an additional effect of Wolbachia density on the sex of diploid wasps. However, since the association of Wolbachia titers with ploidy and sex is correlational, other explanations, including differential Wolbachia proliferation in female ovaries vs male testes [45], cannot be excluded. An explicit test would require manipulation of Wolbachia titers in eggs or early embryos (after sex is determined), which is difficult to perform in practice. Note also that we implicitly assume that the measured Wolbachia titer in adults is proportional to the titer in their embryonic stage, when the endosymbiont acted on host sex determination. This may be a valid assumption given that Landmann et al. [45] found in nematodes that Wolbachia density does not change much during embryonic and early larval developmental stages but proportionally increased with age towards the adult stage.

Studies of Giorgini et al. [9] and Tulgetske [19] have already implied that diploidization and feminization are separate, uncoupled mechanisms in endosymbiontinduced thelytoky. Our results on thelytokous $A$. japonica corroborate this notion and take it a step further to suggest a two-step mechanism for the induction of thelytoky by Wolbachia based on endosymbiont density (Fig. 6): diploidization of the unfertilized egg is followed by feminization, and each step critically relies on a 


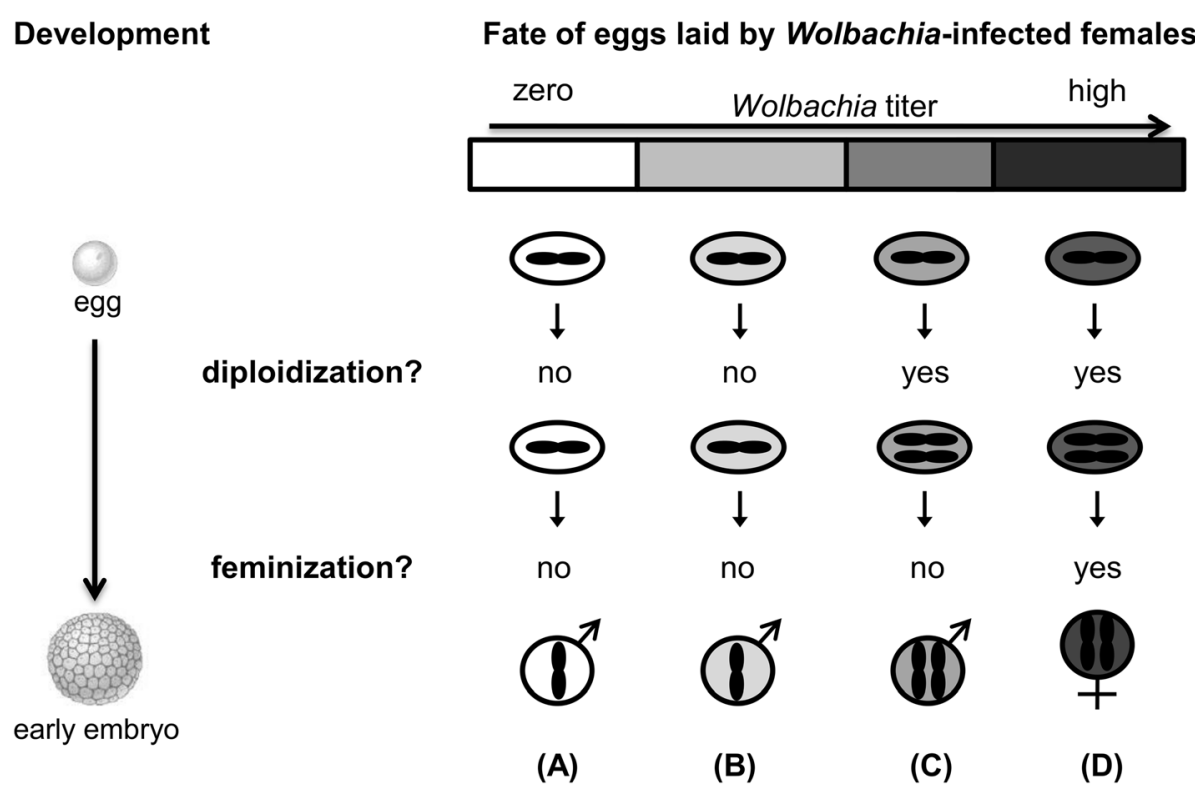

Fig. 6 A two-step model for how endosymbionts induce thelytoky in haplodiploid species. Endosymbionts are considered to induce female development via two distinct steps: diploidization of the egg followed by feminization of the embryo. Each step relies on a certain threshold of endosymbiont titer during early embryonic development when sex is determined. There are four scenarios: (A) Absence of endosymbionts leads to haploid male development; (B) Low endosymbiont titer fails to initiate both diploidization and feminization, leading to development of haploid males as well; (C) Intermediate numbers of endosymbionts trigger diploidization, but are insufficient to induce feminization and result in diploid males; (D) High numbers of endosymbionts cause both diploidization and feminization, leading to diploid female development. Grey shading indicates endosymbiont titer, ranging from zero (white) to high (black)

threshold of endosymbiont titer. In this two-step model, the first step involves diploidization of the unfertilized haploid egg. Absence or a very low density of endosymbionts in eggs would result in haploid male development (Fig. 6A and B). A higher density would result in diploid zygotes that can either develop into males or females (Fig. 6C and D). The second step involves feminization of diploidized embryos, which requires a high Wolbachia titer. If this titer is too low, failure of feminization occurs and embryos develop into diploid males (Fig. 6C). Only a sufficiently high bacterial titer leads to successful diploidization of unfertilized haploid eggs and subsequent feminization of diploid embryos (Fig. 6D). Note that the window of bacterial titer inducing diploidization but not feminization is assumed to be narrow, given that diploid males are rare overall.

Given the evidence of diploidization and feminization being separate processes in A. japonica and two other thelytokous species, there are at least three ways in which endosymbionts could induce thelytoky in haplodiploids. First, the endosymbiont would cause diploidization of haploid eggs, leading to female development following the host's haplodiploid sex determination. Absence or failure of the endosymbiont leads to haploid male development and diploid males cannot occur. This has been suggested to be the case for Wolbachia- induced thelytoky in Leptopilina clavipes [16, 41] and Muscidifurax uniraptor [15]. Second, the endosymbiont feminizes eggs, while egg diploidization is under host control. This appears to apply to Cardinium-induced thelytoky in Encarsia hispida, in which curing of the bacteria leads to diploid rather than haploid male progenies [9]. Finally our results show that Wolbachia causes both diploidization of unfertilized haploid eggs and subsequent feminization of diploid embryos in $A$. japonica. (Partial) curing of thelytoky-inducing endosymbionts can therefore lead to haploid males or diploid males, most likely depending on the individual bacterial load. In addition to A. japonica, this appears to be the case in thelytokous Trichogramma kaykai infected with Wolbachia [19]. Future work needs to clarify whether diploidization and feminization are typically separate processes controlled by the endosymbionts or whether diploidization alone is sufficient to induce thelytoky in some host species. The latter may be difficult to demonstrate experimentally. Indeed, the mechanisms used by the endosymbionts to regulate diploidization and feminization may be highly canalized in most hosts, which would prevent the production of diploid males and generate the false impression of thelytoky induced via diploidization alone when in fact endosymbionts are also regulating feminization. Finally, it is also possible that different 
types of thelytoky induction are associated with specific endosymbiont species, such as Wolbachia, Cardinium and Rickettsia (e.g., [46]).

The realization that endosymbionts can induce thelytoky via diploidization and feminization refines our perspective on the possible constraints imposed by the molecular mechanisms of host sex determination on endosymbiont-induced thelytoky in haplodiploids [21, 47-49]. Two empirically confirmed sex-determination mechanisms have been documented in the Hymenoptera, Complementary Sex Determination (CSD), reported from over 60 hymenopteran species across most major superfamilies [47, 49, 50], and maternal effect genomic imprinting sex determination [51], thus far only described for the parasitoid wasp Nasonia [52]. Under CSD, individuals hemi- or homozygous at one or several loci develop into males, whereas heterozygous individuals develop into females. Hence, CSD has been considered to be incompatible with endosymbiont-induced thelytoky in which egg diploidization occurs via gamete duplication, because homozygous diploid eggs would develop into males rather than females [50,53]. However, this view has to be reconsidered given our findings that both diploidization and feminization are regulated by Wolbachia in A. japonica. Indeed even under CSD, thelytoky via gamete duplication can occur, given the active feminization of diploid eggs by the endosymbiont regardless of the allelic state at the CSD locus. The same holds for the second sex determination mechanism known in hymenopterans, maternal effect genomic imprinting. Under genomic imprinting sex determination, female development depends on activation of the transformer gene in the zygote by a trans factor that is likely transcribed from the parental genomes [52, 54]. This sex determination mechanism could constrain the evolution of thelytoky, as femaleness depends on a parentally contributed chromosome complement (for a detailed discussion see [11]). However, as for CSD, such constraints are released if endosymbionts regulate feminization in addition to diploidization.

Further studies are needed on the interaction between sex determination and endosymbiont-induced thelytoky in A. japonica and other species. This will help to clarify whether or not molecular sex determination mechanisms constrain endosymbiont-induced thelytoky in haplodiploids. They also provide new opportunities for improving our knowledge of the yet poorly-understood sex determination mechanisms in haplodiploid insects. Endosymbiont-induced thelytoky and host sex determination may be subject to tight co-evolution, where one party might have to overcome the constraints set by the other party. It is therefore not unlikely that a completely novel sex determination mechanism, other than CSD or maternal effect genomic imprinting has evolved in $A$. japonica and other species, as a result of Wolbachia infection pressure. Further studies that manipulate endosymbiont titer by antibiotic treatment in combination with measuring gene expression of known sex determination genes may be very informative on the mechanisms of sex manipulation by endosymbionts.

\section{Conclusions}

In this study, we found regular occurrence of diploid males in a Wolbachia-induced thelytokous species. Different approaches were taken to understand the underlying causes of this interesting phenomenon. Wolbachia density in the adult stage was shown to be correlated with the sex and ploidy of individual wasps. An introgression experiment indicated that diploid males were caused by elements encoded by the thelytokous genome. These results led us to propose a two-step mechanism for explaining the occurrence of diploid males: during early embryo development the endosymbiont induces thelytoky via diploidization and feminization. Each step appears to rely on a specific Wolbachia density. This challenges the common view that molecular sex determination mechanisms constrain endosymbiont-induced thelytoky in haplodiploids.

\section{Availability of supporting data}

Data for this study are available at Dryad repository doi: http://dx.doi.org/10.5061/dryad.sk805, and other datasets supporting the results of this article are provided with Additional files.

\section{Additional files}

Additional file 1: Figure S1. Flow cytometric DNA-histograms of a representative (a) haploid male, (b) diploid male and (c) diploid female in the thelytokous KG strain of A. japonica. The $y$-axis depicts the number of nuclei, and the $x$-axis the fluorescence intensity on a log scale, which converts to ploidy as indicated with the $n$-value. An excitation wave length of $488 \mathrm{~nm}$ and a band pass filter of $585 \mathrm{~nm}$ were used to detect propidium iodide fluorescence.

Additional file 2: Figure S2. PCR assay of Wolbachia infection status among haploid males, diploid males and diploid females of the untreated thelytokous KG strain of Asobara japonica, using Wolbachiaspecific wsp gene primers.

\section{Abbreviation}

CSD: Complementary sex determination.

\section{Competing interests}

The authors declare that they have no competing interests.

\section{Authors' contributions}

WJM and LWB wrote the manuscript, commented by all co-authors at all stages. WJM, LVdZ and LWB conceived and designed the experiments. WJM performed the experiments. WJM, TS and BAP analyzed the data. LWB, LvdZ, BAP, BW and TS contributed reagents/materials/analysis tools. All authors read, advised on revisions, and approved the final manuscript. 


\section{Acknowledgements}

We thank Rogier Houwerzijl and Peter Hes for assistance with culturing; Gemma Kulk of Ocean Ecosystems and Anita Kram of Molecular Cell Biology for use of flow cytometers; Ken Kraaijeveld and Barbara Reumer for supplying A. japonica strains, and Elzemiek Geuverink for discussions. This work was supported by TOP grant (no. 854.10.001) of the Netherlands Organization for Scientific Research (NWO) to LWB, a NWO Veni grant no. 863.09.001 to TS and a Horizon Breakthrough (no. 935.19.006) and NGl Zenith (no. 935.11.04) grant from the Netherlands Genomics Initiative to BAP. TS was also supported by the Swiss National Science Foundation (FNS grant PP00P3_139013)

\section{Author details}

${ }^{1}$ Evolutionary Genetics, Groningen Institute for Evolutionary Life Sciences, University of Groningen, Groningen, The Netherlands. ${ }^{2}$ Department of Ecology and Evolution, University of Lausanne, Lausanne, Switzerland. ${ }^{3}$ Laboratory of Genetics, Wageningen University, Wageningen, The Netherlands.

Received: 26 January 2015 Accepted: 29 April 2015

Published online: 12 May 2015

\section{References}

1. Bell G. The masterpiece of nature: the evolution and genetics of sexuality. London: University of California Press; 1982.

2. Normark BB. The evolution of alternative genetic systems in insects. Annu Rev Entomol. 2003;48:397-423.

3. Beukeboom LW, Ellers J, van Alphen JJM. Absence of single-locus complementary sex determination in the braconid wasps Asobara tabida and Alysia manducator. Heredity. 2000;84:29-36.

4. Lattorff HMG, Moritz RFA, Fuchs S. A single locus determines thelytokous parthenogenesis of laying honeybee workers (Apis mellifera capensis). Heredity. 2005;94:533-7.

5. Schwander T, Vuilleumier S, Dubman J, Crespi BJ. Positive feedback in the transition from sexual reproduction to parthenogenesis. Proc R Soc Biol Sci. 2010;277:1435-42.

6. Sandrock C, Vorburger C. Single-locus recessive inheritance of asexual reproduction in a parasitoid wasp. Curr Biol. 2011;21:433-7.

7. Werren JH. Biology of Wolbachia. Annu Rev Entomol. 1997;42:587-609.

8. Werren J, Baldo L, Clark ME. Wolbachia: master manipulators of invertebrate biology. Nat Rev Microbiol. 2008;6:741-51.

9. Giorgini M, Monti M, Caprio E, Stouthamer R, Hunter MS. Feminization and the collapse of haplodiploidy in an asexual parasitoid wasp harboring the bacterial symbiont Cardinium. Heredity. 2009;102:365-71.

10. Kageyama D, Narita S, Watanabe M. Insect sex determination manipulated by their endosymbionts: incidences, mechanisms and implications. Insects. 2012;3:161-99.

11. Ma W-J, Vavre F, Beukeboom LW. Manipulation of arthropod sex determination by endosymbionts: diversity and molecular mechanisms. Sex Dev. 2014;8:59-73.

12. Vorburger $\mathrm{C}$. Thelytoky and sex determination in the hymenoptera: mutual constraints. Sex Dev. 2014;8:50-8.

13. Whiting PW. Selective fertilization and sex-determination in Hymenoptera. Science. 1933;78:537-8

14. Stouthamer R, Kazmer DJ. Cytogenetics of microbe-associated parthenogenesis and its consequences for gene flow in Trichogramma wasps. Heredity. 1994;73:317-27.

15. Gottlieb Y, Zchori-Fein E, Werren JH, Karr TL. Diploidy restoration in Wolbachiainfected Muscidifurax uniraptor (Hymenoptera: Pteromalidae). J Invertebr Pathol. 2002:81:166-74.

16. Pannebakker BA, Beukeboom LW, van Alphen JJM, Brakefield PM, Zwaan BJ. The genetic basis of male fertility in relation to haplodiploid reproduction in Leptopilina clavipes (Hymenoptera: Figitidae). Genetics. 2004;168:341-9.

17. Duron O, Bouchon D, Boutin S, Bellamy L, Zhou L, Engelstädter J, et al. The diversity of reproductive parasites among arthropods: Wolbachia do not walk alone. BMC Biol. 2008;6:27

18. Cordaux R, Bouchon D, Gréve P. The impact of endosymbionts on the evolution of host sex determination mechanisms. Trends Genet. 2011:27:332-41.

19. Tulgetske GM. Investigations into the mechanisms of Wolbachia-induced parthenogenesis and sex determination in the parasitoid wasp, Trichogramma. California: University of California, PhD thesis; 2010
20. Reumer BM, van Alphen JJM, Kraaijeveld K. Occasional males in parthenogenetic populations of Asobara japonica (Hymenoptera: Braconidae): low Wolbachia titer or incomplete coadaptation? Heredity. 2012;108:341-6.

21. Ma W-J, Pannebakker BA, Beukeboom LW, Schwander T, van de Zande L. Genetics of decayed sexual traits in a parasitoid wasp with endosymbiontinduced asexuality. Heredity. 2014;113:424-31.

22. O'Neill SL, Karr TL. Bidirectional incompatibility between conspecific populations of Drosophila simulans. Nature. 1990;348:178-80.

23. Zchori-Fein E, Gottlieb Y, Kelly SE, Brown JK, Wilson JM, Karr TL, et al. A newly discovered bacterium associated with parthenogenesis and a change in host selection behavior in parasitoid wasps. Proc Natl Acad Sci USA. 2001;98:12555-60.

24. Negri I, Franchini A, Gonella E, Daffonchio D, Mazzoglio PJ, Alma A. Unravelling the Wolbachia evolutionary role: the reprogramming of the host genomic imprinting. Proc R Soc B. 2009;276:2485-91.

25. Lu P, Bian G, Pan X, Xi Z. Wolbachia induces density-dependent inhibition to dengue virus in mosquito cells. PLoS Negl Trop Dis. 2012;6, e1754.

26. Dedeine F, Vavre F, Fleury F, Loppin B, Hochberg ME, Boulétreau M. Removing symbiotic Wolbachia bacteria specifically inhibits oogenesis in a parasitic wasp. Proc Natl Acad Sci USA. 2001;98:6247-52.

27. Pannebakker BA, Loppin B, Elemans CPH, Humblot L, Vavre F. Parasitic inhibition of cell death facilitates symbiosis. Proc Natl Acad Sci USA. 2007:104:213-5.

28. Ma W-J, Kuijper B, de Boer JG, van de Zande L, Beukeboom LW, Wertheim $B$, et al. Absence of complementary sex determination in the parasitoid wasp genus Asobara (Hymenoptera: Braconidae). Plos One. 2013;8, e60459.

29. Mitsui H, van Achterberg K, Nordlander G, Kimura MT. Geographical distributions and host associations of larval parasitoids of frugivorous Drosophilidae in Japan. J Nat Hist. 2007;41:1731-8.

30. Murata Y, Ideo S, Watada M, Mitsui H, Kimura MT. Genetic and physiological variation among sexual and parthenogenetic populations of Asobara japonica (Hymenoptera: Braconidae), a larval parasitoid of drosophilid flies. Eur J Entomol. 2009;106:171-8.

31. Ma W-J, Pannebakker BA, Zande Lvd, Schwander T, Wertheim B, Beukeboom LW (2015). Data from: Diploid males support a two-step mechanism of endosymbiont-induced thelytoky in a parasitoid wasp. Dryad Digital Repository. doi:10.5061/dryad.sk805.

32. Aljanabi SM, Martinez I. Universal and rapid salt-extraction of high quality genomic DNA for PCR-based techniques. Nucleic Acids Res. 1997;25:4692-3.

33. Kraaijeveld K, Reumer BM, Mouton L, Kremer N, Vavre F, van Alphen JJM. Does a parthenogenesis-inducing Wolbachia induce vestigial cytoplasmic incompatibility? Naturwissenschaften. 2011;98:175-80.

34. Ruijter JM, Ramakers C, Hoogaars WMH, Karlen Y, Bakker O, van den Hoff $M J B$, et al. Amplification efficiency: linking baseline and bias in the analysis of quantitative PCR data. Nucleic Acids Res. 2009;37, e45.

35. Tuomi JM, Voorbraak F, Jones DL, Ruijter JM. Bias in the Cq value observed with hydrolysis probe based quantitative PCR can be corrected with the estimated PCR efficiency value. Methods. 2010;50:313-22.

36. Ramakers C, Ruijter JM, Deprez RHL, Moorman AFM. Assumption-free analysis of quantitative real-time polymerase chain reaction (PCR) data. Neurosci Lett. 2003:339:62-6.

37. Ma WJ. Evolutionary genetics of Wolbachia-induced parthenogenesis in the parasitoid Asobara japonica. Groningen: University of Groningen. PhD thesis; 2014

38. Crawley MJ. The R, book. Chichester: John Wiley \& Sons Ltd; 2007.

39. Hothorn T, Bretz F, Westfall P. Simultaneous inference in general parametric models. Biometrical J. 2008;50:346-63.

40. Van der Kooi CJ, Schwander T. On the fate of sexual traits under asexuality. Biol Rev. 2014;89:805-19.

41. Pannebakker BA, Pijnacker LP, Zwaan BJ, Beukeboom LW. Cytology of Wolbachia-induced parthenogenesis in Leptopilina clavipes (Hymenoptera: Figitidae). Genome. 2004;303:299-303.

42. Heath BD, Butcher RD, Whitfield WG, Hubbard SF. Horizontal transfer of Wolbachia between phylogenetically distant insect species by a naturally occurring mechanism. Curr Biol. 1999:9:313-6.

43. Sugimoto TN, Ishikawa Y. A male-killing Wolbachia carries a feminizing factor and is associated with degradation of the sex-determining system of its host. Biol Lett. 2012:8:412-5.

44. Narita S, Kageyama D, Nomura M, Fukatsu T. Unexpected mechanism of symbiont-induced reversal of insect sex: feminizing Wolbachia continuously acts on the butterfly Eurema hecabe during larval development. Appl Environ Microbiol. 2007;73:4332-41. 
45. Landmann F, Foster JM, Slatko B, Sullivan W. Asymmetric Wolbachia segregation during early Brugia malayi embryogenesis determines its distribution in adult host tissues. PLoS Negl Trop Dis. 2010;4, e758.

46. Adachi-Hagimori T, Miura K, Stouthamer R. A new cytogenetic mechanism for bacterial endosymbiont-induced parthenogenesis in Hymenoptera. Proc R Soc Biol Sci. 2008;275:2667-73.

47. Asplen MK, Whitfield JB, de Boer JG, Heimpel GE. Ancestral state reconstruction analysis of hymenopteran sex determination mechanisms. J Evol Biol. 2008;22:1762-9.

48. Gottlieb Y. Check out these males. Heredity. 2009;103:1-2

49. Heimpel GE, de Boer JG. Sex determination in the Hymenoptera. Annu Rev Entomol. 2008;53:209-30.

50. Van Wilgenburg E, Driessen G, Beukeboom LW. Single locus complementary sex determination in Hymenoptera: an "unintelligent" design? Front Zool. 2006;3:1.

51. Dobson SL, Tanouye MA. Evidence for a genomic imprinting sex determination mechanism in Nasonia vitripennis (Hymenoptera; Chalcidoidea). Genetics. 1998;149:233-42.

52. Verhulst EC, Beukeboom LW, van de Zande L. Maternal control of haplodiploid sex determination in the wasp Nasonia. Science. 2010;328:620-3.

53. Cook JM. Sex determination in the Hymenoptera: a review of models and evidence. Heredity. 1993;71:421-35.

54. Verhulst EC, Lynch JA, Bopp D, Beukeboom LW, van de Zande L. A new component of the Nasonia sex determining cascade is maternally silenced and regulates transformer expression. Plos One. 2013;8, e63618.

\section{Submit your next manuscript to BioMed Central and take full advantage of:}

- Convenient online submission

- Thorough peer review

- No space constraints or color figure charges

- Immediate publication on acceptance

- Inclusion in PubMed, CAS, Scopus and Google Scholar

- Research which is freely available for redistribution 